\title{
Estudo da cultura organizacional e inovação em uma empresa metalúrgica na
}

\section{percepção dos colaboradores}

\author{
Study of organizational culture and innovation in a metallurgical company in the employees' \\ perception
}

Estudio de la cultura organizacional e innovación en una empresa metalúrgica en la percepción de los empleados

\section{Resumo}

A inovação tem sido tratada como tema relevante nas organizações, onde cada vez mais obter vantagens competitivas tornou-se uma forma de se manter no mercado. Um dos pilares que permitem este processo é a cultura organizacional onde a participação e a interação entre as pessoas e os departamentos favorecem este processo. Neste sentido o estudo busca mapear e analisar como a cultura organizacional em uma empresa de pequeno porte do ramo metalúrgico pode influenciar no processo de inovação. A metodologia utilizada foi a descritiva a partir de um estudo de caso, por meio de abordagem qualitativa e quantitativa, como instrumentos de coleta de dados, aplicaram-se questionário a todos os colaboradores da empresa e realizou-se entrevista semiestruturada com seus diretores. O resultado aponta como positiva as diretrizes tomadas pela organização.

Palavras-chave: Inovação; Conhecimento; Quebra de paradigmas; Estratégia competitiva.

\begin{abstract}
Innovation has been treated as a relevant issue in organizations, where increasingly obtaining competitive advantages has become a way to remain in the market. One of the pillars that allow this process is the organizational culture where participation and interaction between people and departments favor this process. In this sense, the study seeks to map and analyze how the organizational culture in a small company in the metallurgical industry can influence the innovation process. The methodology used was descriptive from a case study, through a qualitative and quantitative approach, as data collection instruments, a questionnaire was applied to all employees of the company and a semistructured interview was carried out with its directors. The result points out as positive the guidelines taken by the organization.
\end{abstract}

Keywords: Innovation; Knowledge; Breaking paradigms; Competitive strategy.

\section{Resumen}

La innovación ha sido tratada como un tema relevante en las organizaciones, donde la obtención cada vez más de ventajas competitivas se ha convertido en una forma de permanecer en el mercado. Uno de los pilares que permiten este proceso es la cultura organizacional donde la participación e interacción entre personas y departamentos favorece este proceso. En este sentido, el estudio busca mapear y analizar cómo la cultura organizacional en una pequeña empresa de la industria metalúrgica puede influir en el proceso de innovación. La metodología utilizada fue descriptiva a partir de un estudio de caso, mediante un enfoque cualitativo y cuantitativo, como instrumentos de recolección de datos, se aplicó un cuestionario a todos los empleados de la empresa y se realizó una entrevista semiestructurada con sus directores. El resultado señala como positivos los lineamientos tomados por la organización. Palabras clave: Innovación; Conocimiento; Rompiendo paradigmas; Estrategia competitiva. 


\section{Introdução}

A cultura organizacional e a inovação não são temas novos. Pelo contrário, elas vêm acontecendo desde os primórdios da humanidade. A necessidade de manter e criar um diferencial competitivo entre as organizações tornou-se cada vez mais relevante, onde evidencia-se o uso do capital humano para a criação e gerenciamento do conhecimento. Desenvolver novos produtos, processos e/ou serviços e a busca por maiores produtividades tem se tornado prática comum e indispensável nas organizações.

Para que uma empresa possa ser considerada inovadora, a inovação precisa ser um valor em si, compartilhado e disseminado como prática diária, em todos os níveis e setores organizacionais e não somente nos departamentos de pesquisa e desenvolvimento, os chamados P\&D (Vidal; Virgillito, 2011).

As organizações de pequeno porte comumente sofrem com a concorrência em relação às grandes empresas, em especial, devido ao difícil acesso a know-how e à baixa força competitiva principalmente quanto aos custos, pessoal, equipamentos e capital (Souza et al., 2015). O cenário de concorrência intensa traz problemas organizacionais para essas empresas e dificulta que permaneçam no mercado, forçando-as à desenvolver novas metodologias na busca de suprir tal desvantagem (Amato Neto, 2008; Cosentino; Vidal; Virgillito, 2011; Bannier; Zahn, 2012).

Uma gestão inovadora e eficiente encontra meios de potencializar conhecimentos disponíveis na organização, melhorando o desempenho no atingimento das metas, cada vez mais exigentes ou até aumentar a motivação dos colaboradores envolvidos, com foco no desenvolvimento organizacional.

Os temas abordados neste estudo tem signifivativa relevância, devido a sua contemporaneidade, servindo como fonte de consulta para futuras pesquisas, bem como contribuindo para uma análise estratégica da empresa em relação ao entendimento e á sua cultura para a inovação.

O objetivo geral desta pesquisa consiste em identificar e analisar, por meio da percepção dos colaboradores e diretores da empresa "W", de que forma a cultura organizacional influência nos processos de inovação da organização, mapeando os pontos positivos e os pontos a serem melhorados.

O resultado dessa análise trará resposta para o seguinte problema de pesquisa: de que forma a cultura organizacional da empresa "W" influencia no processo de inovação? A organização "W" é uma empresa de médio porte, de acordo com a classificação do SEBRAE em relação ao número de empregados (Sebrae, 2018).

\section{Referencial Teórico}

\subsection{Cultura Organizacional}

Segundo Daft (1999) a cultura de uma organização nasce a partir de um líder antecessor que é o responsável por desencadear ideias e valores que serão seguidos e executados dentro da organização. $\mathrm{O}$ autor também fala sobre as duas questões cruciais da cultura dentro das empresas: a integração interna e adaptação externa. Conforme Senhoras (2007), a cultura organizacional trata-se de um estratégico marco conceitual utilizado na administração, uma vez que permite à organização encontrar a sua identidade coletiva de modo a propiciar a criação de significações e mecanismos eficientes de coordenação, comunicação e aprendizagem entre os colaboradores internos, de modo a contribuir para a melhoria da performance organizacional.

Schein (2009) caracteriza a cultura organizacional através de um conceito estrutural em três níveis, a saber: artefatos (estruturas e processos organizacionais visíveis); valores (estratégias, objetivos e filosofias); e pressupostos (que se referem a crenças, percepções e pensamentos, muitas vezes inconscientes). De acordo com o autor, a cultura de um grupo pode ser um padrão de suposições básicas compartilhadas, aprendidas a medida em que solucionavam problemas de adaptação externa e de integração interna, tendo funcionado bem o suficiente para ser repassado aos novos membros (Schein, 2009). 
De acordo com Silva e Fadul (2010), a cultura organizacional abrange um conjunto de valores que se refletem em artefatos visíveis, tais como a linguagem, roupas, mitos e histórias, bem como abrange também as interações entre os indivíduos e grupos, com suas características próprias que os distinguem dos demais.

As pessoas se transformam constantemente, de acordo com o meio em que estão e a necessidade de adequação às demandas dos grupos dos quais se inserem, a cultura organizacional desempenha significante influência na (re)constituição da identidade pessoal desses indivíduos introduzidos no contexto institucional. Essa influência admite a construção da identidade organizacional e uma ideia de integralidade à pessoa, possibilitando-o a edificação da sua imagem em um processo complexo e dinâmico. (MicheL, 2014; Paula, Pinto, Lobato, Mafra, 2014; Resende, Paula, 2011).

Segundo Schein (2010) a cultura organizacional seria vista como resultado da conceituação gerencial; símbolos e significados que seriam transmitidos pela alta direção da organização, sendo reforçados no âmbito de sistemas de incentivos coerentes. O mesmo autor propõe uma visão mais voltada para a primeira abordagem de Morgan, destacando que a cultura organizacional seria composta por variáveis que assumiriam como base dois elementos centrais: a) um elemento informal, criado pelos indivíduos com suas subculturas ou visões; e, b) um elemento formal, ou seja, a cultura oficial da empresa.

Na visão de Hofstede, Hofstede e Minkov (2010) a cultura organizacional como fator contingencial adquiriu um patamar tal qual o da estrutura, enquanto modelo de gestão e estratégia. Este fator, cuja definição já exploramos bem, pode ser também percebido na forma como as empresas se apresentam no mercado e se posicionam frente a concorrência (DE Oliveira, Beuren 2009). Para Crozatti (1998) faz-se necessário que "as crenças e valores culturais da organização sejam movimentados juntamente com os processos, em busca de uma congruência com os valores externos"

A cultura organizacional é ainda vista como uma ferramenta estratégica à produtividade e sucesso das empresas, comunidades e mesmo de um país. Como um conceito, a inovação é, cada vez mais, considerada um fenômeno multidimensional (não linear), que envolve gestão, processos e mercado, permitindo a geração de valor tangível eintangível (Green, et al, 2015).

Contudo, fica evidente a participação dos líderes proporcionando a cultura organizacional, planejando, organizando, dirigindo e controlando os passos para que se possa obter resultados sólidos para a organização torna-se de extrema relevância para atingimento das metas estabelecidas.

\subsection{Conhecimento Organizacional}

As organizações exercem fundamental importância na criação de contextos que estimulem o compartilhamento do conhecimento, tornando-o um facilitador para o aprendizado coletivo. Segundo Santos e Popadiuk (2010), organizações que incentivam as práticas de disseminação e gestão do conhecimento obtém maiores vantagens competitivas além de agregar valor aos seus colaboradores, estimulando o desejo de se fidelizar a organização.

Na visão de Drucker (2011), as tarefas sociais importantes como: desempenho econômico, proteção do meio ambiente, educação e busca por novos conhecimentos são confiadas à gestão das grandes organizações e projetadas para a perpetuidade, estando, tanto a sociedade moderna, quanto até mesmo a sobrevivência dos indivíduos, dependentes do desempenho das organizações. A gestão do conhecimento organizacional contribui para "a compreensão de como recursos intangíveis podem constituir a base de uma estratégia competitiva" e para "a identificação dos ativos estratégicos que irão assegurar resultados superiores para a empresa no futuro (Fleury e Oliveira Junior, 2001).

A capacidade de uma organização em adquirir, transformar e de aprender novos conhecimentos passa a ser relevante para obtenção da inovação em uma organização. Além de gerar novos conhecimentos, as empresas devem fazer conexões com conhecimentos já existentes facilitando o aumento da sua rede de relacionamento organizacional (Eboli, 2004).

Segundo Santos (2013), demonstra que a criação do conhecimento é a dimensão da gestão do conhecimento que mais 
contribui para a geração da inovação, confirmando assim a relação positiva entre a existência de processos de geração do conhecimento no interior das empresas interagindo com as inovações.

O conhecimento organizacional e o seu aprimoramento passaram a ser uma importante ferramenta no auxílio do aumento da produtividade possibilitando que a organização se torne competitiva. Neste sentido, a Gestão do Conhecimento aliado às estratégias de negócio pode trazer grandes benefícios tanto para o setor público quanto para o setor industrial (Terra, 2005).

Neste sentido, investir no conhecimento organizacional torna-se essencial para que ocorra a ampliação do compartilhamento das informações que contribuem para a inovação. Para isso, avaliar o processo é permitir uma visão ampla das etapas necessárias para o desenvolvimento das metas propostas. O papel deste conhecimento é proporcionar condições para criar, adquirir, organizar, disseminar e processar informações estratégicas e, assim, gerar benefícios para a organização, aumentando a competitividade e evidenciando a empresa no mercado em que atua (Strauhs, 2012).

O conhecimento torna-se aliado nas tomadas de decisões bem como nas estratégias das organizações nas organizações reforçando os pontos como: aquisição, organização, armazenamento e disseminação. Costa Neto e Canuto (2012) corroboraram sobre o entendimento da gestão do conhecimento como um dos principais processos para tomada de decisão no que tange a formação, treinamento e experiência por meio das fases elucidadas na Figura 1.

Figura 1: As revoluções industriais.

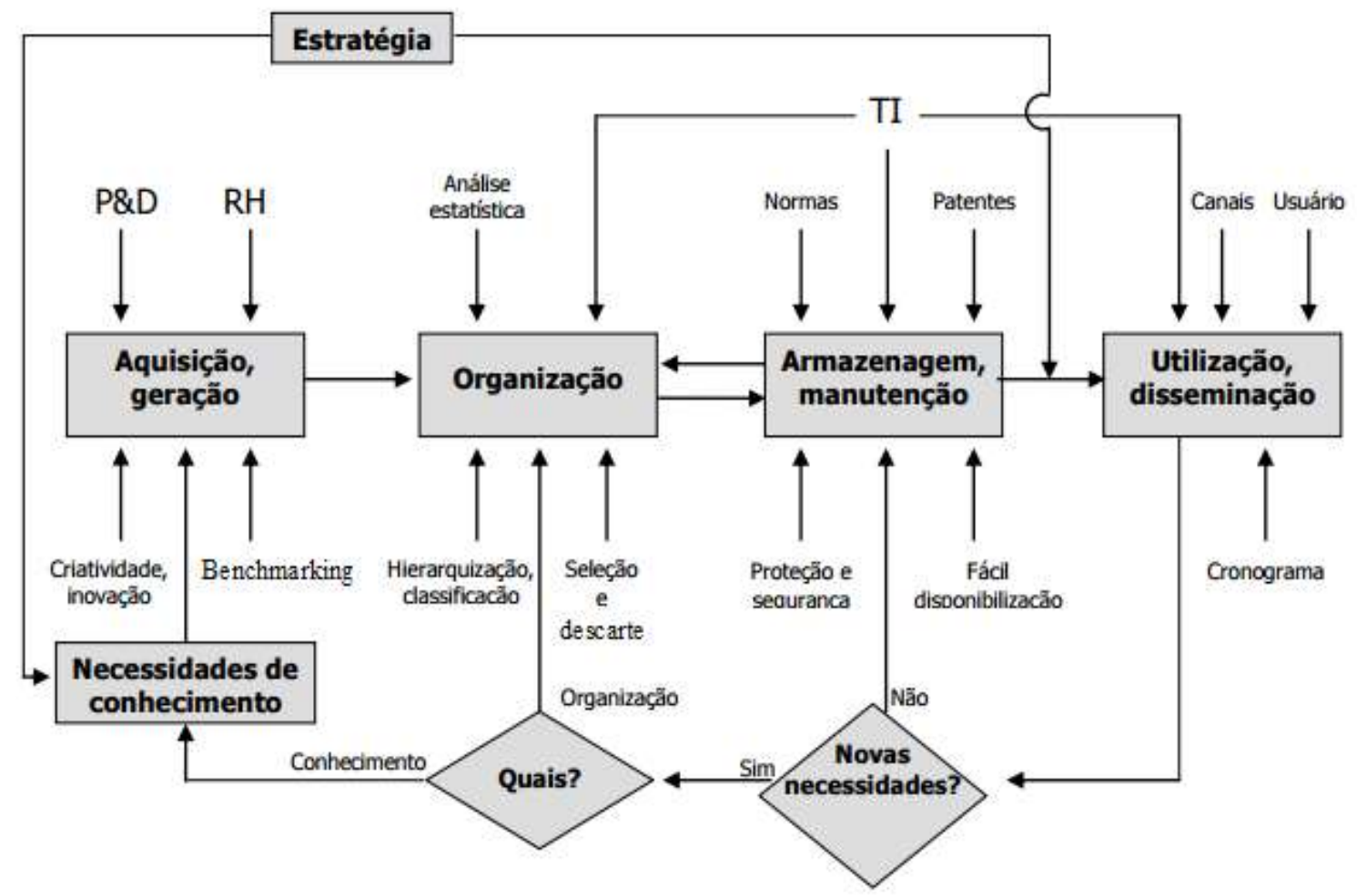

Fonte: Modelo para a Gestão do Conhecimento. Fonte: Costa Neto e Canuto (2012).

Segundo descrito na abordagem de Costa Neto e Canuto (2012) a organização precisa (i) gerar/adquirir conhecimento por meio de contratação de pessoal capacitado e/ou compra de tecnologia; (ii) organização em relação a codificar e hierarquizar os conhecimentos gerados; (iii) armazenamento e manutenção do conhecimento para preservação de novas idéias e (iv) uso e disseminação do conhecimento para todos os envolvidos para melhorar a competitividade organizacional gerando benefícios para a organização promovendo a fidelização de seus clientes. 
Para, Pavlou e El Sawy (2011) indicaram que o conhecimento organizacional pode promover as seguintes capacidades dinâmicas: (1) capacidade de deteç̧ão, definido como a capacidade de identificar, interpretar e buscar oportunidades no ambiente; (2) capacidade de aprendizado, que representa a capacidade de renovar os recursos operacionais existentes com novos conhecimentos; (3) capacidade de integrar, referindo-se à capacidade de introduzir conhecimento individual nos novos recursos operacionais da unidade e (4) capacidade de coordenar ou a capacidade de orquestrar e implantar tarefas, recursos e atividades nos novos recursos operacionais.

\subsection{Inovação Organizacional}

As organizações, como a maioria das empresas, são sistemas complexos em contínua evolução e interação seja com o ambiente interno ou externo. Propor inovações e consequentemente mudanças passa a ser um dos fatores vitais para a sobrevivência da organização na era da competitividade sem fronteiras. A busca por um enfoque abrangente e universal do fenômeno da inovação organizacional torna-se um processo de abrangência setorial que pode e deve ser buscado em qualquer aspecto ou setor da organização (Machado, 2014).

As inovações organizacionais podem ser ocasionadas por diversos fatores, como o envelhecimento e perda de competitividade, a necessidade de atribuir melhores condições de trabalho aos colaboradores, atendimento as necessidades dos clientes, a busca por processos mais eficientes, a busca por novos mercados de atuação entre outros. Uma vez que as características dos ambientes mudam rápida e incessantemente, é fundamental que mudanças organizacionais internas apropriadas sejam continuamente implantadas. Nesse contexto dinâmico, as inovações consistem à base para o alcance da competitividade e da sustentabilidade (Spezamiglio; Galina; Calia, 2016; Vieira; Quadros, 2017).

Uma das limitações ao se considerar as mudanças referentes a inovação organizacional é restringi-la somente ao produto, não levando em consideração os processos, serviços e demais atividades tais como mudanças de mercado, modelos de negócios, métodos organizacionais, fontes de suprimento, entre outras. Criar possibilidades de inovação que proporcionem um crescimento sustentável ao longo do tempo nas organizações passa a ser de extrema relevância para a obtenção de vantagens competitivas (Sambiase; Franklin; Teixeira, 2013).

De acordo com a ótica schumpeteriana, a inovação é um processo contínuo e permanente que rompe com o passado a forma de empregar os recursos produtivos ou comerciais, experimentando uma nova maneira de consumir um produto ou realizar uma atividade (Silva, Paula e Paula, 2017; Sousa e Moreira, 2015; Shafique, 2013). Assim, a inovação pode ser caracterizada pela combinação de recursos novos ou já existentes, a fim de gerar produtos, processos, mercados, métodos de produção ou organização inéditos ou melhorados (Crossan e Apaydin, 2010).

A inovação é movida pela habilidade de estabelecer relações, detectar oportunidades e tirar proveito das mesmas (Tidd, Bessant e Pavitt, 2008). A inovação pode ser considerada como a implementação de um produto, processo, método organizacional e ou de marketing novo ou significativamente melhorado (OCDE, 2005). O Quadro 1 apresenta os quatro tipos de inovações existentes com base nos autores. 
Quadro 1: Tipos de Inovações.

\begin{tabular}{|l|l|}
\hline $\begin{array}{l}\text { Inovação de } \\
\text { Produto }\end{array}$ & $\begin{array}{l}\text { É a introdução de um bem ou serviço novo ou significativamente melhorado no que } \\
\text { concerne a suas características ou usos previstos. Incluem-se melhoramentos significativos } \\
\text { em especificaçães técnicas, componentes e materiais, softwares incorporados, facilidade } \\
\text { de uso ou outras características funcionais. }\end{array}$ \\
\hline $\begin{array}{l}\text { Inovação de } \\
\text { Processo }\end{array}$ & $\begin{array}{l}\text { É a implementação de um método de produção ou distribuição novo ou significativamente } \\
\text { melhorado. Incluem-se mudanças significativas em técnicas, equipamentos e/ou } \\
\text { softwares. }\end{array}$ \\
\hline $\begin{array}{l}\text { Inovação de } \\
\text { Marketing }\end{array}$ & $\begin{array}{l}\text { É a implementação de um novo método de marketing com mudanças significativas na } \\
\text { concepção do produto ou em sua embalagem, no posicionamento do produto, em sua } \\
\text { promoção ou na fixação de preços. }\end{array}$ \\
\hline $\begin{array}{l}\text { Inovação } \\
\text { Organizacional }\end{array}$ & $\begin{array}{l}\text { É a implementação de um novo método organizacional nas práticas de negócios da } \\
\text { empresa, na organização do seu local de trabalho ou em suas relações externas. }\end{array}$ \\
\hline
\end{tabular}

Fonte: Adaptado de OCDE (2005).

Os quatro elementos apresentados no Quadro 1, inovação de produto, inovação de processo, inovação de marketing e inovação organizacional são formas de potencializar as organizações independentemente do seu ramo de atuação bem como do seu porte.

A busca por novas tecnologias, por novas formas de aprendizado possibilitam à organização elaborar e implementar um fluxo de conhecimentos que permeia a interação entre o aprendizado individual e organizacional que consiste em um insumo necessário ao processo inovativo bem como a utilização do know-how adquirido pela organização.

A importância do relacionamento e dos processos de interação na promoção da atividade inovativas se deve ao fato de manterem canais e fluxos de saberes tácitos e explícitos - principalmente do âmbito individual para o organizacional - que propiciam a criação, transformação e compartilhamento do conhecimento permitindo as inovações (Gardim; Cartoni; Caballero, 2011).

Com tal característica, a capacidade de inovar, advém da execução de novas combinações materiais e forças produtivas, ocasionando a ruptura do fluxo circular e a tendência ao desenvolvimento, ou seja, a evolução econômica decorre do processo permanente de "destruição criativa", que permite vantagem competitiva para a organização inovadora (Howaldt, Domanski \& Kaletka, 2016; Schumpeter, 1928).

De acordo com Fauchart e Keilbach (2009) os modelos de aprendizagem oferecem uma base microeconômica para heterogeneidade, que enfatiza de maneira interessante os processos experimentais dinâmicos inerentes a entrada e ao crescimento da empresa no mercado, assumindo desvios sistemáticos que podem surgir no processo de desenvolvimento organizacional.

De modo geral, destaca-se que o efeito das inovações voltadas ao produto também converge para as variáveis de processo, estando geralmente direcionadas à inovações incrementais que visam apenas modificações graduais (Sanches e Machado, 2013).

Concomitantemente, a Eficiência tende para as inovações sustentáveis relacionadas a Ecoinovação, revelando uma preocupação, ainda que tímida, com a redução de custos e o impacto das empresas sobre o meio ambiente (Campos, Santos \& Donadon, 2017).

Segundo Tidd, Bessant e Pavitt (2008), o desenvolvimento de novos produtos é uma capacidade importante, pois o meio ambiente está constantemente mudando; alternâncias no campo socioeconômico criam oportunidades e restrições. O Quadro 2 mostra alguns aspectos que decorrem sobre a aplicação da Inovação organizacional. 
Quadro 2: Aspectos sobre a Inovação Organizacional.

\begin{tabular}{|c|c|}
\hline Aspectos & Descrição \\
\hline $\begin{array}{l}\text { A inovação tende a } \\
\text { aparecer em ambientes } \\
\text { com diversidade de } \\
\text { habilidades e culturas }\end{array}$ & $\begin{array}{l}\text { Grupos de trabalho constituídos por profissionais de diferentes origense } \\
\text { formação tendem a trabalhar num clima mais aberto e mais informado. O sucesso } \\
\text { na inovação estará relacionado às condiços existentes que permitem errar e } \\
\text { assumir riscos. A inovậa nunca é um resultado monolitico. }\end{array}$ \\
\hline $\begin{array}{l}\text { A inovação consistente } \\
\text { pode garantir a liderança } \\
\text { de mercado }\end{array}$ & $\begin{array}{l}\text { No entanto, é preciso estabelecer algumas condiçoses. Primeiro, o processo de } \\
\text { inovação deve começar pelo suporte da direção da empresa que acredita na } \\
\text { mudança como vantagem competitiva. Segundo, a cultura da mudança deve se } \\
\text { espalhar pela empresa por meio da promoção de um ambiente competitivo e } \\
\text { constante avaliação dos avanços alcançados. }\end{array}$ \\
\hline Benchmork & $\begin{array}{l}\text { É preciso escolher um concorrente que servirá de referência e alvo de superaçäo, } \\
\text { o benchmork, incentivando e premiando sempre os profissionais que se } \\
\text { envolveram com mais denodo na tarefa. Em especial deve-se deixar a inovạão } \\
\text { crescer. Novas ideias precisam de patrocinadores e lideres defensores, um misto } \\
\text { de pessoas criativas nas ideias e que não fogem da operação (mantendo as coisas } \\
\text { práticas). }\end{array}$ \\
\hline Foco no cliente & $\begin{array}{l}\text { Um último obstáculo para o éxito no processo de inovação é o conhecimento do } \\
\text { comprador. A ideia aqui é abandonar a tentativa de entendê-lo. O truque é } \\
\text { oferecer ao mercado produtos programáveis ou sujeitos á personalização. A } \\
\text { verdadeira construção de valor acontecerá no momento em que o cliente puder } \\
\text { projetar sua própria versão daquilo que realmente quer. Com isso, fica alterado } \\
\text { completamente o processo usual de agregação de valor via inovậ̃o. Caberá } \\
\text { agora ao cliente um papel importante e até arriscado, pois ele será parte } \\
\text { integrante em todo processo de renovaçäo mudança. }\end{array}$ \\
\hline
\end{tabular}

Fonte: Adaptado de Chiavenato e Sapiro (2003).

Embora o processo de inovação seja cada vez mais vista e compreendida como uma importante maneira de assegurar possíveis vantagens competitivas passa a ser uma maneira mais segura de defender posições estratégicas, bem como obter o sucesso nem é sempre garantido, uma vez que os mercados estão cada vez mais competitivos a acirrados independentemente do ramo ou segmento da organização (Tidd, Bessant \& Pavitt, 2008).

\subsection{Estratégia Competitiva}

Porter (1999) defende a estratégia competitiva resultante de um processo de planejamento sistemático e explícito, capaz de nortear a coordenação e a direção dos diversos departamentos na empresa, proporcionando-lhe vantagem competitiva. E define o processo para formulação da estratégia "como a construção de defesas contra as forças competitivas ou como a descoberta de posições no setor onde as forças são mais fracas" (Porter, 1999) cuja essência consiste na escolha de atividades distintas da maioria dos rivais, com vistas à criação de uma posição denominada pelo autor como "exclusiva e valiosa" (Porter, 1999), capaz de criar valor aos acionistas.

Cada organização busca uma estratégia competitiva que melhor se adere ao seu modelo. No entanto, o que possibilita a construção dessas estratégias de competitividade está ligado a uma visão integradora de seus gestores e ao mercado em que atua (Kim; Mauborgne, 2005).

Proporcionar qualificação aos seus colaboradores passa a ser uma estratégia valorosa uma vez que que o conhecimento é essencial para elevar a força competitiva de uma empresa, potencializando o capital humano em prol do crescimento organizacional, beneficiando não só a empresa bem como a sociedade como um todo, agregando valor também ao nome da organização (Cosentino; Vidal; Virgillito, 2011; Sense, 2011; Maes; Sels, 2014).

Os gestores estão conscientes da nova dinâmica e das restrições de seus relacionamentos com clientes, fornecedores e concorrentes, é importante que estes gerentes sejam aconselhados a examinar o conhecimento da mesma forma que consideram os modelos contábeis, administrativos e comerciais que adotam para os ativos tangíveis e intangíveis da organização, (Fleury E 
Oliveira Junior, 2001).

A estratégia competitiva deve fazer parte da organização. A eficácia tende a aumentar quando as estratégias corporativas são implementadas permitindo assim que haja sinergia tornando mais eficientes as ações criadas pelos colaboradores da organização independentemente do seu nível hierárquico. A Figura 2 identifica alguns dos fatores que envolvem a estratégia competitiva.

Figura 2: Estratégia competitiva.

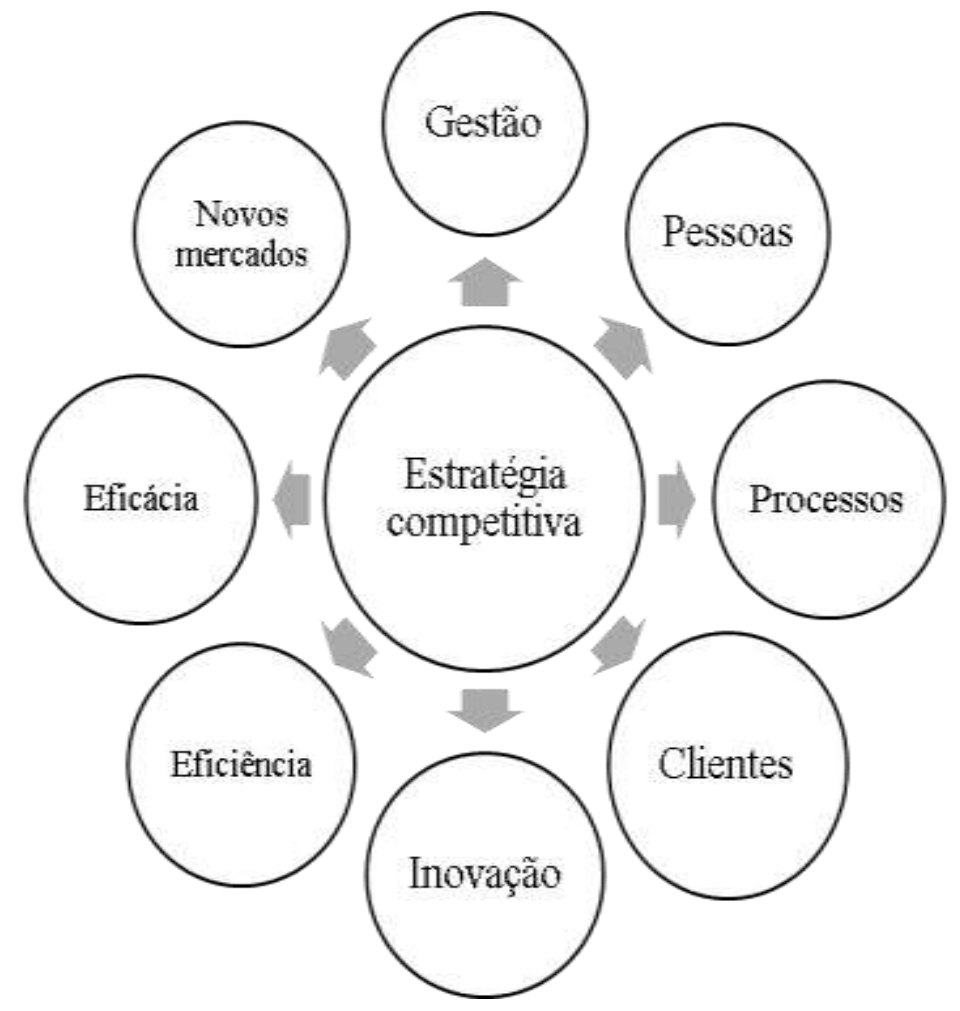

Fonte: Autores.

A Figura 2 ilustra as interações e as sinergias que a estratégia competitiva gera nas organizações, onde torna-se possível o compartilhamento de diversos componentes permitindo assim agregar valor e a construir uma vantagem competitiva sólida para a empresa.

O planejamento estratégico das organizações é caracterizado pela sua estrutura organizacional e possibilidade de mercado (Neis et, al. 2016). A partir do planejamento estratégico, organizações utilizam projetos para geração dos resultados projetados. Projetos são realizados visando à efetivação das estratégias competitivas (Junior et, al, 2011). Comumente, as empresas desenvolvem um portfólio com diferentes projetos, priorizando conforme cenário e oportunidade de mercado (Valle et, al. 2010). A criação de um projeto surge a partir da necessidade ou planejamento estratégico da organização, e o entendimento claro do que se busca é estritamente necessário para projetar metas e escopo para execução (Heldman, 2009).

\section{Metodologia}

Segundo Vergara (2004), as pesquisas descritivas "expõe características de determinada população ou de determinado fenômeno, não tendo como compromisso de explicar os fenômenos que descreve, embora sirva de base para tal explicação".

Gil (2017) acrescenta que algumas pesquisas descritivas vão além da simples identificação da existência de relações entre variáveis, pretendendo determinar a natureza dessa relação. Cita ainda a existência de pesquisas que, "embora definidas 
como descritivas a partir de seus objetivos, acabam servindo mais para proporcionar uma nova visão do problema, o que as aproxima das pesquisas exploratórias".

Quanto aos meios, a presente pesquisa pode ser classificada como um estudo de caso. Ainda segundo Gil (2008) esse tipo de estudo é "caracterizado pelo estudo profundo e exaustivo de um ou de poucos objetos, de maneira a permitir conhecimentos amplos e detalhados".

Para Yin (2014) o estudo de caso, além de ser importante para que se possa compreender os fenômenos sociais complexos, ele "permite uma investigação para se preservar as características holísticas e significativas dos eventos da vida real tais como os processos organizacionais.

Para pesquisas com abordagem qualitativa, Collis e Hussey (2005) informam que a entrevista e a observação são dois dos mais importantes instrumentos para se coletar dados e entendimento da situação a ser estudada.

\section{Resultados e Discussão}

Para contextualização do perfil da orgnização foco do estudo, apresenta-se algumas informações relevantes sobre a empresa. A empresa "W", iniciou suas atividades em julho de 1982 em São Paulo capital e hoje está localizadade em diadema SP, conta com 192 colaboradores, distribuidos em dois turnos nas áreas de: diretoria, gerencia industrial, gerencia comercial, engengaria, produção, qualidade, manutenção, logística, ferramentaria e administrativo.

A empresa "W" trabalha com produto próprio (conectores para componentes eletronicos) e também prestação de serviços nas áreas de injeção de plástico e de alumínio, atuando no ramo metalúrgico no segmento automotivo, elétrico, eletrõnico e também na linha branca. Trata-se de uma empresa familiar onde os pais e os filhos (2), comandam a organização atuando como diretores.

Os colaboradores que responderam ao questionário foram 19 no total, sendo diretores, gerentes, supervisores e lideres de setores, sendo que alguns com 2 a 3 anos de empresa. Esse dado converge com o crescimento da organização nesse espaço de tempo, tendo em vista que a empresa vem se expandindo ao decorrer dos últimos anos por meio de novas parcerias com outras empresas do mesmo segmento.

A organização por diretrizes estabelecidas por seus diretores tem como meta em um ano aumentar a abrangência de seus produtos por meio de novos lançamentos. Para isso está atualizando seu pátio fabril bem como realizando novas contratações na parte administrativa quanto na fabril.

Utilizou-se a escala Likert para mensurar a importância das competências do Engenheiro de Produção associados a processos de inovação. Para Cunha (2007), uma escala tipo Likert é composta por um conjunto de itens e, em relação a cada item, o entrevistado manifesta o seu grau de concordância. Os dados foram coletados entre novembro e dezembro de 2021, através de entrevistas realizadas com colaboradores das empresas.

Cada respondente se manifestou sobre a importância das atividades inovativas referentes a cada uma das dimensões da qualidade da inovação consideradas, de acordo com a escala.

\begin{tabular}{|c|c|c|c|c|}
\hline $\begin{array}{c}\text { Concordo } \\
\text { totalmente }\end{array}$ & Concordo & $\begin{array}{c}\text { Nem concordo } \\
\text { nem discordo }\end{array}$ & Discordo & $\begin{array}{c}\text { Discordo } \\
\text { totalmente }\end{array}$ \\
\hline $\mathbf{5}$ & $\mathbf{4}$ & $\mathbf{3}$ & $\mathbf{2}$ & $\mathbf{1}$ \\
\hline
\end{tabular}

Foram elencadas questões que permitem identificar como se configura a cultura organizacional na empresa "W", no que se refere à formação de um ambiente propício para a inovação, conforme ilustra a Tabela 1. 
Tabela 1: Questões sobre a cultura organizacional

\begin{tabular}{|c|c|c|c|c|c|c|}
\hline Perguntas & $\begin{array}{c}\text { Concordo } \\
\text { totalmente } \\
\%\end{array}$ & $\begin{array}{c}\text { Concordo } \\
\%\end{array}$ & $\begin{array}{c}\text { Nem } \\
\text { concordo } \\
\text { e nem } \\
\text { discordo }\end{array}$ & $\begin{array}{c}\text { Discordo } \\
\%\end{array}$ & $\begin{array}{c}\text { Discordo } \\
\text { totalmente } \\
\%\end{array}$ & Total \\
\hline $\begin{array}{l}1 \text {-A empresa "w" é uma } \\
\text { empresa inovadora? }\end{array}$ & $42 \%$ & $35 \%$ & $23 \%$ & $0 \%$ & $0 \%$ & $100 \%$ \\
\hline $\begin{array}{l}2 \text { - Há uma frequente } \\
\text { rudança em temos de } \\
\text { organização (pessoas) na }\end{array}$ & $17 \%$ & $39 \%$ & $19 \%$ & $25 \%$ & $0 \%$ & $100 \%$ \\
\hline $\begin{array}{c}3 \text { - Faço parte do processo } \\
\text { de inovação da } \\
\text { organização? }\end{array}$ & $62 \%$ & $25 \%$ & $12 \%$ & $1 \%$ & $0 \%$ & $100 \%$ \\
\hline $\begin{array}{l}4 \text { - A empresa estimnla a } \\
\text { busca pelo conhecimento? }\end{array}$ & $36 \%$ & $21 \%$ & $25 \%$ & $9 \%$ & $9 \%$ & $100 \%$ \\
\hline $\begin{array}{l}5 \text { - A empresa permite a } \\
\text { exposição de novas idéias? }\end{array}$ & $39 \%$ & $28 \%$ & $13 \%$ & $15 \%$ & $5 \%$ & $100 \%$ \\
\hline $\begin{array}{c}\text { 6- As atividades são todas } \\
\text { documentadas? }\end{array}$ & $28 \%$ & $29 \%$ & $19 \%$ & $10 \%$ & $14 \%$ & $100 \%$ \\
\hline $\begin{array}{c}7 \text { - A cormunicação dentro } \\
\text { da empresa é clara, objetiva } \\
\text { e documentada }\end{array}$ & $38 \%$ & $52 \%$ & $8 \%$ & $2 \%$ & $0 \%$ & $100 \%$ \\
\hline $\begin{array}{c}8 \text { - A empresa "W" } \\
\text { proporciona um ambiente } \\
\text { familiar? }\end{array}$ & $29 \%$ & $23 \%$ & $19 \%$ & $29 \%$ & $0 \%$ & $100 \%$ \\
\hline $\begin{array}{l}9 \text { - As estratégias } \\
\text { competitivas são coerentes } \\
\text { com a realidade da }\end{array}$ & $31 \%$ & $27 \%$ & $20 \%$ & $12 \%$ & $10 \%$ & $100 \%$ \\
\hline
\end{tabular}

Fonte: Autores.

\section{A percepção dos colaboradores conforme apontado na Tabela 1.}

1 - Os diretores da empresa, afirmam que a inovação está intimanente ligada a cultora da organização, seja por meio dos projetos realizados ou pela valorização de seus colaboradores. Com relação aos colaboradores $77 \%$ concorda que a empresa é inovadora. Pode se constatar que a organização é inovadora, pois são direcionados recursos e esforços para desenvolvertecnologias e oferecer aos clientes novaos modelos de produtos, visando atender as suas necessidades.

2 - Para os diretores a busca por colaboradores qualificados também é relevante e proporciona uma troca de informações entre as pessoas e também deve servir de exemplo para aqueles que não queiram se enquadar no escopo da organização. Para os colaboradores 56\% concordam que há uma relenate troca de colaboradores, porém $44 \%$ não tem opinião sobre o assunto ou são contrários a esta ação, e deve ser levado em consideração pela alta direção da organização. 
3 - Dentre os respondentes, $87 \%$ afirmam se sentir parte do processo de inovação da organização. Para os diretores todos fazem parte do processo de inovação. O processo de inovação na empresa "W" é compartilhado entre todos os colaboradores. Segundo Machado e Barzotto (2012) colocar sobre a interação de diferentes habilidades, percepções e níveis de energia de cada pessoa que está atuando no processoem conjunto estimula o potencial para as inovações.

4 - Um dos fatores relevantes e de destaque pela direção da empresa esta relacionado a questão dos treinamentos que são ministrados dentro e fora da organização na qualificação de seus colaboradores. Para $57 \%$ dos entrevistados a organização se preocupa com a qualificação profissional e $18 \%$ discordam ou não tem interesse em se qualificar profissionalmente.

5 - Ao serem questionados sobre a abertura para a exposição de ideias e sugestões, os diretores expuseram que todos os colaboradores têm a oportunidade de dar ideias e sugestões em todos os setores da empresa. Para $67 \%$ dos colaboradores, concordam com essa colocação e $20 \%$ são contrários a esta afirmação.

6 - Há um entendimento entre os diretores que o processo de documenta da organização deveria ser mais eficiente, o que também é perceptível pelos colaboradores uma vez que 57\% concorda com a afirmação, porém se considerarmos o restante $43 \%$ são contrários a afirmação, o que permite uma atenção especial a este ponto como sendo foco para melhorias futuras.

7 - Segundo os respondentes embora a documentação da organização deva ser melhorada a comunicação entre as pessoas e os departamentos é positiva e bem clara, com 90\% de aprovação pelos entrevistados, sendo o ponto de destaque as atas que são geradas a cada reunião feito dentro ou fora da organização e compartilhada com todos os departamentos, mesmo que seja a título de conhecimento.

8 - Por fim, $52 \%$ dos pesquisados concordou que, para eles, a "W" apresenta um ambiente familiar, em que se sentem confortáveis. $19 \%$ não concordam e nem discordam, 29\% discordam dessa afirmativa. Segundo os diretores, a empresa busca oferecer um ambiente agradável para os funcionários.

9 - Para os diretores a estratégia da organização está adequada e atual ao mercado, as necessidades econômicas e principalmente aos seus clientes. Para seus colaboradores 58\% estão de acordo com as estratégias estabelecidas pela empresa e buscam o atingimento da mesma e até mesmo a superação destas metas estabelecidas. Os colaboradores têm a consciência que deste modo a organização prospera e consequentemente todos que estão nela.

\section{Conclusão}

O objetivo da pesquisa foi identificar e analisar, por meio da percepção dos funcionários e diretores da empresa "W", de que maneira a cultura organizacional pode influenciar nos processos de inovação na empresa.

Quanto aos aspectos de inovação questionados aos colaboradores, verificou-se que reconhecem o esforço da organização para se tornar inovadora, e que a mesma está em constante mudança para o atingimento desta meta, bem como sabem da sua importância neste processo, sendo uma evolução organizacional e pessoal. Acima de tudo, a cultura organizacional presente na empresa estabelece um ambiente de trabalho agradável, em que os colaboradores se sentem 
motivados a colaborar, gerando melhorias nos processos produtivos de uma forma geral.

$\mathrm{Na}$ organização existem metodologias aplicadas e bem-sucedidas bem como algumas que necessitam de aprimoramento e devem ser encaradas como oportunidades de melhorias, sendo que os próprios diretores reconhecem esta vulnerabilidade no processo de inovação organizacional.

Como sugestão de melhorias a serem trabalhadas na cultura, voltadas à inovação, indica-se um olhar atento à questão da comunicação interna. Destaca-se, ainda, que este trabalho poderá cooperar para a análise estratégica da empresa potencializando os pontos positivos da organização, satisfazendo aos seus clientes internos, esternos e aos seus acionistas.

\subsection{Trabalhos futuros}

$\checkmark$ Aplicação da pesquisa em outras organizações com o intuito de analisar quais os seus resultados nestas empresas.

\section{Agradecimentos}

$\checkmark$ Aos nossos familiares pelo apoio, carinho e compreensão.

\section{Referências}

Amato Neto, J. (2013). Productive cooperation network as a competitive advantage for small and medium firms in the Brazilian state of São Paulo. International Journal of Entrepreneurship and Small Business, 5(2), 201-211.

Campos, M. G. D., Santos, D. F. L., \& Donadon, F. A. B.(2017). Impacto dos investimentos em inovação na indústria brasileira. Revista de Gestão Industrial, v. 13 (3), 213-36.

Chiavenato, I., \& Sapiro, A. (2003). Planejamento estratégico: fundamentos eaplicações. Elsevier.

Collis, J., \& Hussey, R. (2005). Pesquisa em administração: um guia prático para alunos de graduação e pós-graduação. Bookman.

Cosentino, H. M., Vidal, P. G., \& Virgillito, S. B. (2011). Gestão de conhecimento e competitividade nas empresas de pequeno porte do setor de comércio exterior de autopeças: um estudo exploratório. RAI Revista de Administração e Inovação, 8(3), 100-119.

Costa, P. L. D. O., \& Canuto, S. A. (2010). Administração com qualidade. Blucher.

Crossan, M. M., A\& paydin, M. (2010). A Multi-Dimensional Framework of Organizational Innovation: A Systematic Review of the Literature. Journal of Management Studies, v. 47 (6), 1154-91.

Crozatti, J. (1998). Modelo de gestão e cultura organizacional: conceitos e interações. Caderno de estudos, 01-20.

Cunha, L. M. A. D. (2007). Modelos Rasch e Escalas de Likert e Thurstone na medição de atitudes (Doctoral dissertation).

Daft, R. L. (1999). Teoria e Projeto das Organizações, 6ed Rio de Janeiro: Editora LTC.

de Oliveira, E. L., \& Beuren, I. M. (2009). Adequação dos controles de gestão às contingências ambientais em empresa familiar do ramo de papel e celulose. Revista de Contabilidade do Mestrado em Ciências Contábeis da UERJ, 14(1), 33-49.

de Oslo, O. M. (2005). Diretrizes para coleta e interpretação de dados sobre inovação. Organização para a Cooperação e Desenvolvimento Econômico.

de Souza, G. H. S., Lima, N. C., de Miranda Coelho, J. A. P., de Oliveira, S. V. W. B., \& Milito, C. M. (2015). A influência das redes de cooperação no desenvolvimento de micro e pequenas empresas (MPES). Desenvolvimento em Questão, 13(31), 259-294.

Eboli, M. (2004). Educação corporativa no Brasil: mitos e verdades. In Educação corporativa no Brasil: mitos e verdades (pp. 278-278).

Fauchart, E., \& Keilbach, M. (2009). Testing a Model of Exploration and Exploitation as Innovation Strategies. Small Business Economics, v. 33, p. 257-272.

Fleury, M. T. L., \& Oliveira Junior, M. (2001). Gestão estratégica do conhecimento: integrando aprendizagem, conhecimento e competências. São Paulo: Atlas.

Gardim, N., Cartoni, D., \& Caballero, S. (2011). A disseminação do conhecimento no apoio à inovação e sustentabilidade organizacional: a importância dos portais corporativos. Gestão da Sustentabilidade Organizacional: Desenvolvimento de Ecossistemas Colaborativos. 1ed. Campinas: Cedet, 81-100.

Gil, A. C. (2008). Métodos e técnicas de pesquisa social. (6a ed.), Ediitora Atlas SA.

GIL, A. C. (2017). Pós-Graduação-Metodologia-Como Elaborar Projetos de Pesquisa-Cap 2. 
Green, R., Agarwal, R., Brown, P. J., Bajada, C., \& Shashnov, M. (2015). Innovation Capability and the Food Beverage and agri-business sectors.

Heldman, K. (2009). Gerência de projetos: guia para o exame oficial do PMI. Elsevier.

Hofstede, G., Hofstede, G. J., \& Minkov, M. (2005). Cultures and organizations: Software of the mind (Vol. 2). Mcgraw-hill.

Howaldt, J., Domanski, D., \& Kaletka, C. (2016). Social Innovation: Towards a New Innovation Paradigm. Revista de Administração Mackenzie, São Paulo, v. 17 , n. 6 , p. $20-44$.

Junior, L. J. M., \& Plonski, G. A. (2011). Gestão de projetos em empresas no Brasil: abordagem “tamanho único"? Gest. Prod. 18(1).

Kim, W. C., \& Mauborgne, R. (2005). La estrategia del océano azul (p. 23). Elsevier.

Machado, D. D. P. N., \& da Costa Barzotto, L. (2012). Ambiente de inovação em instituição hospitalar. RAI Revista de Administração e Inovação, 9(1), 51-80.

Machado, R. E. (2014). Influência do capital intelectual na capacidade absortiva e na inovação.

Maes, J., \& Sels, L. (2014). SMEs' radical product innovation: The role of internally and externally oriented knowledge capabilities. Journal of Small Business Management, 52(1), 141-163.

Michel, A. (2014). The mutual constitution of persons and organizations: An ontological perspective on organizational change. Organization Science, 25(4), 1082-1110.

Neis, D. F., Pereira, M. F., \& Maccari, E. A. (2016). Strategic Planning Process and Organizational Structure: Impacts, Confluence and Similarities. Brazilian Business Review.

Paula, A. V. D., Pinto, L. B., Lobato, C. B. D. P., \& Mafra, F. L. N. (2014). Desenho também é coisa séria: desvelando o" funcionário padrão" da sociedade capitalista moderna no desenho animado bob esponja calça quadrada. RAM. Revista de Administração Mackenzie, $15,45-71$.

Pavlou, P. A., \& El Sawy, O. A. (2011). Understanding the elusive black box of dynamic capabilities. Decision sciences, 42(1), $239-273$.

Porter, M. E.(1999). Competição: Estratégias Competitivas Essenciais. Campus.

Resende, F. G., \& de Paula, A. V. (2011). Influência da cultura organizacional na (re) construção da identidade dos trabalhadores: um estudo de caso em uma empresa de tratamento de resíduos no sul de Minas Gerais. Psicologia: teoria e prática, 13(3), 124-138.

Sambiase, M. F., Franklin, M. A., \& Teixeira, J. A. (2013). Inovação para o desenvolvimento sustentável como fator de competitividade para as organizações: um estudo de caso Duratex. RAI Revista de Administração e Inovação, 10(2), 144-168.

Sanches, P. L. B., Machado, A. G. C. (2013). Estratégias de Inovação e RBV: Evidências em uma Empresa de Base Tecnológica. Revista de Administração e Inovação, São Paulo, v. 10, n. 4, p. 183-207.

Santos, A. C. B. (2013). O Impacto da Gestão do Conhecimento e da Inovação na Performance das Empresas do Distrito de Leiria (Doctoral dissertation).

Santos, A. E. M. D., \& Popadiuk, S. (2010). A gestão do conhecimento e a capacidade de competição.

SCHEIN, E. H. (2009). Cultura organizacional e liderança/Edgar H. Schein. Atlas.

Schein, E. H. (2010). Organizational culture and leadership (Vol. 2). John Wiley \& Sons.

Schumpeter, J. (1928). The Instability of Capitalism. The Economic Journal, 38, 361-386.

SEBRAE (2018). Critérios e conceitos para classificação de empresas. <http://www.sebrae.com.br/uf/goias/indicadores-das-mpe/classificacao-empresarial>.

Senhoras, E. M. (2007). Culture in hospital organizations and cultural policies for coordinating communication and learning. Elect J Commun Inf Innov Health, 1(1), 45-55.

Sense, A. J. (2011). The project workplace for organizational learning development. International Journal of Project Management, $29(8), 986-993$.

Shafique, M. (2013). Thinking Inside The Box? Intellectual Structure Of The Knowledge Base Of Innovation Research (1988-2008). Strategic Management Journal, 34, 62-93.

Silva, C. H. P. D., Paula, V. M. F. D., \& Paula, V. A. F. D. (2017). Marcas Inovadoras: Como os Consumidores Percebem a Inovação. Revista Brasileira de Marketing, 16, 83-97.

Silva, L. P. D., \& Fadul, É. (2010). A produção científica sobre cultura organizacional em organizações públicas no período de 1997 a 2007: um convite à reflexão. Revista de Administração Contemporânea, 14, 651-669.

Sousa, E. G. D. S., \& Moreira, M. F. (2015). Inovações em Software: Como as Empresas do Distrito Federal as Gerenciam? Organizações em Contexto, São Bernardo do Campo, 11, 279-306.

Spezamiglio, B. D. S., Galina, S. V. R., \& Calia, R. C. (2016). Competitividade, inovação e sustentabilidade: uma inter-relação por meio da sistematização da literatura. REAd. Revista Eletrônica de Administração (Porto Alegre), 22, 363-393.

Strauhs, F. R., Pietrovski, E. F., Santos, G. D., Carvalho, H. G., Pimenta, R. B., \& Penteado, R. S. (2012). Gestão do conhecimento nas organizações. Curitiba: Aymará.

Terra, J. C. C. (2005). Gestão do conhecimento: o grande desafio empresarial. 
Research, Society and Development, v. 10, n. 9, e53210918588, 2021

(CC BY 4.0) | ISSN 2525-3409 | DOI: http://dx.doi.org/10.33448/rsd-v10i9.18588

Tidd, J., Bessant, J., \& Pavitt, K. (2008). Gestão da Inovação. Bookman.

Valle, A. B., Soares, C. A. P., Finocchio JR, J., \& Silva, L. S. F. (2010). Fundamentos do Gerenciamento de Projetos. Rio de Janeiro: FGV.

Vergara, S. C. (2004). Projetos e relatórios de pesquisa em administração. Atlas.

Vieira, G., \& Quadros, R. (2017). Organização para inovação: Integrando estratégia, estrutura e processos de gestão. Desafio online, 5(2).

Yin, R. K. (2015). Estudo de Caso-: Planejamento e métodos. Bookman editora. 